\title{
INIERE-SPACQE
}

\section{A INTERDISCIPLINARIDADE ENTRE PSICOLOGIA HOSPITALAR, REABILITAÇÃO E EDUCAÇÃO NO TRABALHO COM CRIANÇAS COM LESÃO CEREBRAL}

\section{THE INTERDISCIPLINARITY BETWEEN HOSPITAL PSYCHOLOGY, REHABILITATION AND EDUCATION IN WORK WITH CHILDREN WITH BRAIN INJURY}

\section{INTERDISCIPLINARIDAD ENTRE LA PSICOLOGÍA HOSPITAL, REHABILITACIÓN Y EDUCACIÓN EN EL TRABAJO CON NIÑOS CON LESIÓN CEREBRAL}

\section{Carolina Alves Rezende Alcântara}

Psicóloga pela Universidade Federal da Bahia - UFBA. Especialista em Terapia AnalíticoComportamental Infantil pelo Instituto Brasiliense de Análise do Comportamento e Especialista em Psicologia Hospitalar pelo Conselho Federal de Psicologia. Psicóloga Hospitalar na Rede Sarah Hospitais de Neurorreabilitação - São Luís/MA. carolinaar@sarah.br/karol_allves@yahoo.com.br

Recebido para avaliação em 22/02/2017; Aceito para publicação em 31/10/2017.

\section{RESUMO}

Este trabalho busca apontar possíveis contribuições da atuação como psicóloga hospitalar de reabilitação para o processo de aprendizagem escolar e escolarização inclusiva de crianças com lesão cerebral, considerando relato de experiência das atividades desenvolvidas no Hospital Sarah de Neurorreabilitação, unidade de São Luís, Maranhão. Pauta-se, portanto, na perspectiva que evidencia a interdisciplinaridade entre as áreas da psicologia hospitalar, reabilitação e educação. Entende-se que os profissionais da psicologia hospitalar no contexto de reabilitação podem se constituir um pilar na rede de apoio necessária ao processo de escolarização inclusiva, ampliando a compreensão sobre o desenvolvimento global para os diversos agentes envolvidos na reabilitação.
\end{abstract}

Palavras-chave: Psicologia Hospitalar; Reabilitação; Educação; Infância.

\section{ABSTRACT}

This work seeks to point out possible contributions of the performance as a rehabilitation hospital psychologist for the school learning process and inclusive schooling of children with brain injury, considering experience reports of the activities developed at the Sarah Network of Neurorehabilitation Hospital, São Luís, Maranhão. Therefore, it is based on the perspective that evidences the interdisciplinary between the areas of hospital psychology, rehabilitation and education. It is understood that the professionals of hospital psychology in the context of rehabilitation can constitute a pillar in the support network necessary to the process of inclusive schooling, broadening the understanding on the global development for the diverse agents involved in the rehabilitation.

Keywords: Hospital Psychology; Rehabilitation; Education; Infancy.

\section{RESUMEN}

Este trabajo busca identificar posibles contribuciones de actuar como un psicólogo de 
Dossiê: Diálogos interdisciplinares em Psicologia da Educação

|A interdisciplinaridade entre Psicologia Hospitalar, reabilitação e educação no trabalho com crianças com lesão cerebral|

\section{| Carolina Alves Rezende Alcântara |}

rehabilitación del hospital para el proceso de aprendizaje escolar y la educación inclusiva de los niños con lesión cerebral, teniendo en cuenta experiencia en el informe de las actividades desarrolladas en el Hospital de Neurorehabilitación Sarah, São Luís unidad, Maranhão. Pautase por lo que desde la perspectiva que pone de relieve las áreas interdisciplinares de psicología de la salud, rehabilitación y educación. Se entiende que los profesionales de la psicología de la salud en el contexto de rehabilitación pueden ser un pilar en la red de apoyo necesario para el proceso escolarización inclusiva, ampliando la comprensión del desarrollo global de los diversos actores involucrados en la rehabilitación.

Palabras clave: Psicología de la Salud; Rehabilitación; La Educación; Infancia.

\section{INTRODUÇÃO}

O objetivo deste trabalho é apontar possíveis contribuições da atuação como psicóloga hospitalar de reabilitação para o processo de aprendizagem escolar e inclusão de crianças com lesão cerebral. Trata-se de um relato de experiência no qual serão descritas práticas de atuação que apontam para a interdisciplinaridade entre psicologia hospitalar, reabilitação e educação, no trabalho com crianças com lesão cerebral.

O artigo será estruturado de modo a apontar especificidades no trabalho com crianças com lesão cerebral e sua demanda por serviços de apoio ao processo de escolarização inclusiva, considerando-se características do desenvolvimento dos sujeitos em questão. Em seguida, será apresenta uma contextualização da política de inclusão no Brasil e legislação que sustenta a atuação de profissionais que atuam com crianças com necessidade educacionais especializadas. Por fim, será apresentado um relato de experiência, a partir do trabalho como psicóloga hospitalar na Rede Sarah de Hospitais de Neurorreabilitação, com conjunto de práticas de atuação voltadas para a área, além de considerações que podem contribuir para o campo de pesquisa e prática profissional dirigida a crianças com lesão cerebral.

\section{ASPECTOS DO DESENVOLVIMENTO EM CRIANÇAS COM LESÃO CEREBRAL}

No que se refere à lesão cerebral na infância tem-se que estas podem ser categorizadas como congênitas ou adquiridas. As lesões congênitas são aquelas cujos danos ocorrem no período pré-natal ou perinatal e as lesões adquiridas ocorrem em fases posteriores do desenvolvimento. Dentre os fatores potencialmente determinantes de uma lesão cerebral irreversível, os mais comumente observados são infecções do sistema nervoso, hipóxia (falta de oxigênio) e traumas de crânio. Na prática clínica com crianças 
Dossiê: Diálogos interdisciplinares em Psicologia da Educação

|A interdisciplinaridade entre Psicologia Hospitalar, reabilitação e educação no trabalho com crianças com lesão cerebral|

\section{| Carolina Alves Rezende Alcântara |}

com lesão cerebral, diagnósticos mais comuns incluem a paralisia cerebral e traumatismo crânio encefálico.

No trabalho com crianças com lesão cerebral, algumas desordens no desenvolvimento podem ser observadas. Estas incluem alterações no desenvolvimento cognitivo e neuropsicológico, comprometimento motor, alterações visuais e auditivas, epilepsia, problemas de comportamento, dentre outras alterações (REDE SARAH, 2015). Há, portanto, um processo de aprendizagem em eixos do desenvolvimento no qual uma variável orgânica interatua com variáveis ambientais na emissão de respostas pela criança.

O psicólogo hospitalar no contexto de reabilitação de crianças com lesão cerebral pode contribuir com o processo de avaliação do desenvolvimento global desses sujeitos, em especial no que se refere aos eixos cognitivo, neuropsicológico e comportamental, identificando áreas de maior potencialidade para estimulação, áreas que demandarão maior apoio ao longo do desenvolvimento e favorecendo a construção de estratégias para o manejo no cotidiano e estimulação.

Dentro do processo de análise do desenvolvimento, de acordo com Duran e Goodman (2000), as evidências conclusivas sobre a associação entre alterações cerebrais e problemas de comportamento na infância são recentes. Os autores afirmam que crianças com alterações cerebrais têm probabilidade pelo menos duas vezes maior de desenvolverem problemas psiquiátricos, independentemente de outros fatores.

Estudos apontam ser comum a persistência de problemas de comportamento após uma lesão cerebral, havendo também dados que sugerem que a frequência de alterações comportamentais na criança, anteriores à lesão, são preditores e aumentam a frequência desse problema após a ocorrência de traumatismo crânio encefálico (YLVISAKER; FEENEY, 2008).

Nas crianças com lesão cerebral adquirida, os problemas de comportamento tendem a ser mais comuns e mais graves na medida em que as crianças são mais novas. Relacionam-se com características do desenvolvimento cognitivo e com características da lesão cerebral (YLVISAKER; FEENEY, 2008). Tem-se, portanto, um processo no qual a lesão cerebral e suas consequências implicam em mudanças fundamentais na família, sendo justificadas as propostas terapêuticas que possam incidir na atenuação desses problemas ou no desenvolvimento de repertórios de enfrentamento a eles (FORMAN, 2008).

Problemas de comportamento comuns apresentados por crianças com lesão cerebral incluem desinibição, agressividade, respostas de imaturidade, inflexibilidade, depressão, retraimento social, hiperatividade, oposicionismo (FEENEY; YLVISAKER, 
Dossiê: Diálogos interdisciplinares em Psicologia da Educação

|A interdisciplinaridade entre Psicologia Hospitalar, reabilitação e educação no trabalho com crianças com lesão cerebral|

\section{| Carolina Alves Rezende Alcântara |}

2006). No entanto, entende-se que esses comportamentos são multideterminados, havendo a interferência de variáveis orgânicas, ambientais imediatas, culturais no seu processo de determinação e manutenção.

Catroppa e Anderson (2009), em um trabalho sobre reabilitação de crianças que sofreram lesão cerebral traumática, apontam que alterações comuns pós-lesão incluem hiperatividade, dificuldades na atenção e memória, nas habilidades psicomotoras, funções executivas e na aprendizagem de conteúdos acadêmicos. Todas essas alterações podem implicar em mudanças no funcionamento global da criança e na sua participação no contexto da escola. Esses autores destacam ainda que novos arranjos para inclusão no ambiente escolar devem ser pensados e destacam estratégias de adaptação de tarefas, flexibilização de tempo, eliminação de distratores e uso de recursos externos para suporte.

Prigatano e Gray (2007), em uma pesquisa para avaliar fatores que interferem no nível de estresse parental de famílias cujos filhos sofreram lesão cerebral, apontam que a despeito da gravidade da lesão, as dificuldades no desempenho escolar configuram importante fonte de preocupação e indicam a necessidade de ser estabelecido um modelo educacional que contemple a necessidade desses sujeitos. Os autores sinalizam a importância de uma comunicação efetiva com a equipe da escola com objetivo de reduzir conflitos entre professores, famílias e alunos e abordam a participação de profissionais especializados.

Os dados supracitados ilustram, portanto, que há possibilidade de apresentação de características específicas no desenvolvimento que justificarão intervenções específicas de reabilitação e análise criteriosa do desenvolvimento voltada para promoção de estratégias que qualifiquem a vida das crianças com lesão cerebral.

\section{A REDE SARAH E ATENDIMENTO A CRIANÇAS COM LESÃO CEREBRAL}

A Rede Sarah de Neurorreabilitação é uma referência no Brasil no atendimento de problemas neurológicos em crianças e adultos. Consiste de nove unidades e atende pacientes provenientes de todo o país. $\mathrm{Na}$ infância, grande parte da população atendida apresenta o diagnóstico de paralisia cerebral ou lesão cerebral traumática. As crianças e adolescentes são atendidos através de diversas modalidades de tratamento por uma equipe interdisciplinar de saúde (BRAGA; ROSSI; COLE, 2010). A unidade de São Luís, Maranhão, localiza-se no bairro Monte Castelo e conta com os Programas de Neurorreabilitação em Lesão Medular, Reabilitação Neurológica e Ortopedia, onde são 
Dossiê: Diálogos interdisciplinares em Psicologia da Educação

|A interdisciplinaridade entre Psicologia Hospitalar, reabilitação e educação no trabalho com crianças com lesão cerebral|

\section{| Carolina Alves Rezende Alcântara |}

admitidos adultos e crianças.

Os principais diagnósticos relacionados à Reabilitação Infantil tratados na unidade de São Luís são: paralisia cerebral; sequelas de lesão encefálica; sequelas de lesão medular e de traumatismo cranioencefálico; mielomeningocele; artrogripose; pé torto congênito e neurogênico; variação da normalidade (pé plano fisiológico, joelho valgo, joelho varo); Legg Calvé Perthes; sequela de poliomielite; luxação congênita de quadril e lesão de plexo braquial obstétrico (REDE SARAH, 2015).

Além do atendimento hospitalar, esta unidade possui um Centro Comunitário, aberto à população, composto por Biblioteca, Brinquedoteca e Espaço de Criação, além de ampla área verde. Nesse espaço, a reabilitação dos pacientes ganha um novo componente beneficiando-se da interface com a comunidade local.

O Hospital SARAH São Luís dispõe do Programa de Educação e Prevenção de Acidentes da Rede SARAH, que elabora e ministra palestras para estudantes das redes pública e privada de ensino. No programa da reabilitação infantil, a população mais frequentemente atendida é composta por crianças e adolescentes com lesão cerebral.

A motivação para o desenvolvimento desse trabalho situa-se em uma prática com crianças com lesão cerebral, na qual um dos objetivos como psicóloga hospitalar está voltado para a compreensão do funcionamento global da criança, avaliação de seu desenvolvimento cognitivo, neuropsicológico e comportamental, análise de como essas características do desenvolvimento interferem na vida familiar, escolar, e social dessa criança, e na proposição de estratégias de reabilitação. Estas são baseadas na estruturação de um manejo ambiental, propostas educativas com familiares e outros agentes sociais, e discussão de estratégias complementares com outras especialidades tais como neuropediatras, psiquiatras infantis, pediatras, educadores físicos, pedagogos e outros profissionais da equipe interdisciplinar. Objetiva-se que as estratégias traçadas qualifiquem a vida da criança e família e sua participação nos ambientes do cotidiano. Na infância, um dos espaços sociais e de aprendizagem mais significativos é a escola, sendo necessário serem pensadas estratégias que favoreçam o processo de inclusão.

\section{CONTEXTUALIZAÇÃO DA POLÍTICA DE INCLUSÃO: escolarização,} atendimento educacional especializado e serviços de apoio

De acordo com Alcântara (2013), ao realizar um panorama histórico da política de inclusão no Brasil, a Educação Especial no Brasil pode ser compreendida como uma 
Dossiê: Diálogos interdisciplinares em Psicologia da Educação

|A interdisciplinaridade entre Psicologia Hospitalar, reabilitação e educação no trabalho com crianças com lesão cerebral|

\section{| Carolina Alves Rezende Alcântara |}

construção histórica, um resultado de movimentos constantes que remetem necessariamente à formação da sociedade brasileira. Nesse sentido, Jannuzzi (2006) aponta para o inter-relacionamento entre a educação oferecida para o aluno com deficiência e as transformações sociais, políticas e culturais pelas quais passou a sociedade brasileira.

Mazzotta (2005) afirma que a história da Educação Especial no país tem início ainda no século XIX com a organização de serviços para atendimento a cegos, surdos e deficientes mentais e físicos. A partir da década de 60, a administração pública veio compreender que a Educação Especial se configura como um tópico dos deveres do Estado com a sociedade. Desde então, foram propostas políticas educacionais que tinham como pauta a Educação Especial. Neste momento histórico, a Educação Especial era, em linhas gerais, restrita às empresas que prestavam serviços às classes altas, restando à outra parcela da sociedade, apenas cuidados medicalizantes (MAZZOTTA, 2005).

Esse panorama, com algumas transformações contextuais, permaneceu no país até a década de 80 , quando se iniciou uma discussão mais sistemática sobre a responsabilidade do poder público frente a educação das pessoas com deficiência, atrelando deveres de Estado a direitos humanos.

Bueno (2004) analisa que a expansão e democratização na Educação Especial responderam mais a um processo segregador da sociedade que possuía um meio social carente e pouco estimulador, do que à democratização da Educação. Devido a esse fator, a Educação Especial foi ampliando seu público, partindo de cegos e surdos, e incorporando deficientes mentais, deficientes físicos, crianças com distúrbios mentais graves, até acolher pessoas com distúrbios de conduta, de linguagem e de aprendizagem. Embora, no Brasil, as pessoas com distúrbios de linguagem e de aprendizagem sempre ficaram em pauta na discussão se seriam ou não público da Educação Especial

No final da década de 80 e início da década de 90 do século XX, se observa um processo de maior articulação entre Educação Especial e políticas públicas. Envolve-se aqui a Constituição de 1988, a Declaração de Salamanca de 1994, a Lei de Diretrizes e Bases da Educação de 1996, entre outros diversos dispositivos que culminaram na recente Política Nacional de Educação Especial na perspectiva da Educação Inclusiva, de 2008 (CARVALHO, 2006).

De acordo com Alonso (2014), a Educação inclusiva compreende a educação especial dentro da escola regular, com meta de transformar a escola em um espaço para todos. A proposta estaria centrada em favorecer a diversidade na medida em que considera que todos os alunos podem ter necessidades especiais em algum momento de sua vida 
Dossiê: Diálogos interdisciplinares em Psicologia da Educação

|A interdisciplinaridade entre Psicologia Hospitalar, reabilitação e educação no trabalho com crianças com lesão cerebral|

\section{| Carolina Alves Rezende Alcântara |}

escolar.

Considerando a inclusão de crianças e adolescentes que apresentem necessidades especiais ao longo do seu processo de aprendizagem, compreende-se que há necessidades que interferem de maneira significativa no processo de aprendizagem e que exigem uma atitude educativa específica da escola como, por exemplo, a utilização de recursos e apoio especializados para garantir a aprendizagem de todos os alunos.

A proposta de Educação Inclusiva no Brasil está ancorada em legislação específica que norteia o sistema educacional. Nesse sentido, o Plano Nacional de Educação (PNE 2011-2020), regulamentação mais recente, estabelece a nova função da educação especial e aborda o atendimento educacional especializado. Este apoio de caráter especializado prevê suporte ao aluno acerca de suas necessidades educacionais especiais, tais como o ensino de linguagens e códigos específicos de comunicação e sinalização, no caso da deficiência visual e auditiva; mediação para o desenvolvimento de estratégias de pensamento, no caso da deficiência intelectual; adaptações do material e do ambiente físico, no caso da deficiência física; estratégias diferenciadas para adaptação e regulação do comportamento, no caso do transtorno global; ampliação dos recursos educacionais e/ou aceleração de conteúdos para altas habilidades.

Dentro do processo de educação inclusiva tem-se ainda sido discutidas as redes de apoio adicionais ao processo de aprendizagem. Alonso (2014) aponta que a família compõe a rede de apoio como a instituição primeira e significativamente importante para a escolarização dos alunos, sendo fonte de informações para o professor sobre a criança e destacando-se, dessa forma, a necessidade de uma relação de cooperação entre escola e família. Os profissionais da área de saúde que trabalham com o aluno, como fisioterapeutas, psicopedagogos, psicólogos, fonoaudiólogos ou médicos, também compõem a rede, podendo esclarecer necessidades e sugerir alternativas que possam ser benéficas no processo de aprendizagem. No atendimento a crianças com lesão cerebral, os profissionais da equipe interdisciplinar de reabilitação podem funcionar como importante elo e compor a rede de apoio que favorece a escolarização inclusiva.

\section{O TRABALHO DA PSICOLOGIA HOSPITALAR NO CONTEXTO DE REABILITAÇÃO E SUA INTERFACE COM A PSICOLOGIA DA EDUCAÇÃO}

As crianças que apresentam um quadro de lesão cerebral e são atendidas no Hospital Sarah são acompanhadas através de um modelo de atendimento individualizado e 
Dossiê: Diálogos interdisciplinares em Psicologia da Educação

|A interdisciplinaridade entre Psicologia Hospitalar, reabilitação e educação no trabalho com crianças com lesão cerebral|

\section{| Carolina Alves Rezende Alcântara |}

com propostas de reabilitação baseadas no contexto e na participação familiar. Ao iniciar acompanhamento na unidade, as crianças são avaliadas por uma equipe interdisciplinar de saúde e, considerando-se as demandas trazidas pela família, são traçadas metas para o programa de reabilitação. Essas metas podem incluir áreas diversas do desenvolvimento e participação social da criança. Um eixo de intervenção comum refere-se às estratégias voltadas para o processo de escolarização, seja na etapa de inserção ou na proposição de adaptações ao longo da participação escolar em distintas etapas do desenvolvimento da criança.

Dentro do contexto de atendimento e atenção a crianças com lesão cerebral na Rede Sarah Hospitais de Neurorreabilitação, os profissionais que compõem a equipe interdisciplinar de saúde têm suas práticas guiadas e ancoradas por uma proposta de reabilitação baseada no contexto, o Método Sarah de tratamento (BRAGA; PAZ JÚNIOR, 2008). Dentro dessa proposta, a equipe desenvolve sua atividade profissional em conjunto com a família e buscando intervenções que façam sentido no contexto e comunidade na qual a criança está envolvida. Nesta perspectiva, entende-se que o desenvolvimento da criança é mediado a partir da interação com as pessoas próximas e estabelecem-se cinco princípios básicos de atendimento na oferta de um serviço de qualidade à população em atendimento.

Dessa forma, pretende-se: criar um programa individualizado apropriado ao estágio de desenvolvimento específico da criança, que seja lúdico, usando materiais simples e integrando atividades de diferentes especialidades em uma mesma tarefa para facilitar a aprendizagem; desenvolver um programa fundamentado em objetivos viáveis e realistas, baseado no prognóstico motor, neuropsicológico e na capacidade de comunicação da criança; garantir a integração da família, da criança e dos membros da equipe de reabilitação; contextualizar o programa do desenvolvimento e integrar a criança ou adolescente à comunidade; dar suporte à família com grupos de apoio e reuniões informativas sobre o problema da criança.

No programa de reabilitação, as crianças são acompanhadas junto às famílias por uma dupla interdisciplinar de profissionais que auxilia na condução do programa de reabilitação e articula a indicação de avaliações e apoio de outros membros da equipe. Dentro do plano de reabilitação, a participação em grupos de estimulação se configura como uma ferramenta de suporte às propostas específicas do programa. Nos grupos, são desenvolvidas atividades de interesse das crianças participantes que se articulam às metas específicas de trabalho de cada criança. Objetiva-se desenvolver atividades que expressem 
Dossiê: Diálogos interdisciplinares em Psicologia da Educação

|A interdisciplinaridade entre Psicologia Hospitalar, reabilitação e educação no trabalho com crianças com lesão cerebral|

\section{| Carolina Alves Rezende Alcântara |}

interesses particulares do sujeito e possam, por este motivo, serem mais sucedidas quanto à generalização das habilidades adquiridas.

Nesse contexto de atuação interdisciplinar de reabilitação, ocorre a prática da psicologia hospitalar. A despeito da possibilidade do programa de estimulação ser desenvolvido através da participação em grupos e modelos de aprendizagem que incluem os pares, a análise do desenvolvimento de cada criança ocorre de forma individualizada, a fim de serem identificadas áreas de potencialidade e áreas que demandarão maior apoio ao longo do programa.

A psicóloga hospitalar tem a sua prática na equipe voltada para esta meta. Objetivase ainda uma avaliação das variáveis que atuam no processo de aprendizagem da criança, de modo a promover no ambiente modificações específicas que estejam voltadas para a instauração e fortalecimento de repertório em prol do processo de aprendizagem acadêmica. As atividades específicas a serem desenvolvidas por este profissional incluem avaliação do neurodesenvolvimento; avaliação neuropsicológica e cognitiva; avaliação comportamental; avaliação psicopedagógica; condução de grupos de estimulação; promoção de visitas domiciliares e escolares; elaboração de documentos sobre o desenvolvimento da criança.

Dentre os objetivos específicos, voltados para o processo de escolarização, contemplados nas intervenções realizadas tem-se favorecer adaptação curricular e manejo de comportamento, considerando-se os dados do desenvolvimento global da criança e orientar diagnóstico. A natureza das atividades anteriormente referidas é específica e difere dos processos avaliativos e de estimulação que ocorrem no âmbito da escola.

Os atendimentos desenvolvidos por este profissional podem ocorrem de forma, individual ou em grupos, contando ainda com demais integrantes da equipe interdisciplinar de saúde.

Um caso clínico que ilustra as propostas de atendimento desenvolvidas com a participação da psicologia hospitalar refere-se ao processo de intervenção de DICM, 13 anos de idade. A criança apresenta o diagnóstico de Paralisia Cerebral do tipo tetraplegia espástica.

Longitudinalmente fora avaliado quanto ao desenvolvimento, sendo observado bom potencial global, e dificuldades específicas, em especial no eixo das habilidades perceptivo motoras. Essa alteração trazia repercussão significativa na qualidade e execução das atividades escolares, considerando as dificuldades para o grafismo, desorganização espacial, além de ritmo lento para as atividades que envolviam a escrita. 
Dossiê: Diálogos interdisciplinares em Psicologia da Educação

|A interdisciplinaridade entre Psicologia Hospitalar, reabilitação e educação no trabalho com crianças com lesão cerebral|

\section{| Carolina Alves Rezende Alcântara |}

Avaliou-se ainda haver maior dificuldade para o desenvolvimento das funções executivas que envolvem habilidades específicas de planejamento, organização e controle gerencial na execução de tarefas. O quadro comportamental da criança também se configurou como uma variável que interferia no seu processo de aprendizagem pedagógica e estimulação cognitiva. Apresentava defasagem no desenvolvimento de habilidades sociais, em especial habilidades de assertividade, empatia e desenvoltura social.

Todas as características cognitivas, neuropsicológicas e comportamentais previamente citadas interferiam no processo de aprendizagem acadêmica. Foram discutidas com a família estratégias que podiam favorecer a reabilitação através da estimulação mais contextual e estimulação em ambientes sociais com pares. No contexto escolar, foram construídas possibilidades de intervenção junto à equipe da escola com uso de estratégias que incluíam fragmentação de atividades mais complexas para favorecer organização e planejamento; construção de roteiros para atividades de estimulação com discriminação para a criança das etapas envolvidas na resolução dos problemas propostos; elaboração e construção conjunta com a criança de acordos prévios sobre as atividades que seriam realizadas nos momentos de estimulação; organização de arranjos de aprendizagem com mediação mais próxima de um adulto ou par mais capaz nos momentos de estimulação; adaptação do conteúdo acadêmico, respeitando a fase do desenvolvimento atual da criança; flexibilização do tempo para atividades que envolviam a escrita, bem como estratégias de redução desta; uso de ferramentas complementares para atividades de escrita (computador, tablet). Em conjunto ainda com a equipe escolar, fora ainda proposto um treino contextualizado de estimulação de habilidades sociais.

\section{CONSIDERAÇÕES FINAIS}

As atividades desenvolvidas e destacadas ao longo do relato de experiência ilustram contribuições possíveis da equipe interdisciplinar de neurorreabilitação e psicologia hospitalar para o processo de escolarização inclusiva de crianças que apresentam lesão cerebral, visando qualificar o processo de aprendizagem de crianças que apresentam alterações cognitivas, comportamentais e neuropsicológicas como proposta complementar de intervenção na escola, dentro de uma abordagem de reabilitação baseada no contexto dos sujeitos.

Dessa forma, entende-se que os profissionais da psicologia hospitalar no contexto de reabilitação podem se constituir um pilar na rede de apoio necessária ao processo de 
Dossiê: Diálogos interdisciplinares em Psicologia da Educação

|A interdisciplinaridade entre Psicologia Hospitalar, reabilitação e educação no trabalho com crianças com lesão cerebral|

\section{| Carolina Alves Rezende Alcântara |}

escolarização inclusiva, através de uma proposta de atuação que evidencia a interdisciplinaridade envolvida no trabalho com crianças com lesão cerebral.

Um dos aspectos relevantes para reflexão de profissionais de saúde que atuam com crianças e inclusão escolar refere-se à noção de que a participação dos profissionais e processo de atuação através da sua prática profissional não implica em desresponsabilizar ou desautorizar a equipe escolar do processo de avaliação e proposição de estratégias para o aluno. Pensar em uma avaliação sobre o desenvolvimento de uma criança ultrapassa a identificação de déficits e estratégias compensatórias. Envolve um processo de conhecimento mais global sobre a criança, no qual seus interesses, habilidades positivas e potencialidades precisam ser continuamente pensados, e não são atribuição exclusiva da atuação de profissionais de saúde. Nesse processo de conhecimento global da criança todos os agentes estão implicados e podem ser ferramentas para modificações e qualificação da aprendizagem nos contextos naturais dos quais a criança participa.

De mesmo modo, a participação dos profissionais de saúde no processo de escolarização inclusiva não descaracteriza a necessidade de investimento na educação e processo de formação de professores. São intervenções que podem ser pensadas como complementares, porém nunca em uma perspectiva de que uma ação exclua a outra.

\section{REFERÊNCIAS}

ALCÂNTARA, R. L. S. A ordem do discurso na educação especial. São Luís: EDUFMA, 2013.

ALONSO, D. Os desafios da educação inclusiva: foco nas redes de apoio. Disponível em: <https://novaescola.org.br/conteudo/554/os-desafios-da-educacao-inclusiva-foconas-redes-de-apoio $>$. Acesso em: 15 jul. 2014.

ALVES, P. A ecologia do desenvolvimento humano: experimentos naturais e planejados. Psicol. Reflex. Crit., Porto Alegre, v. 10, n. 2, p. 369-373, 1997. Disponível em: $<$ http:/ / www.scielo.br/scielo.php?script=sci arttext\&pid=S0102-79721997000200013 >.

Acesso em: 15 jul. 2014.

BRAGA, L. W.; ROSSI, L.; COLE, M. Criar uma idiocultura para promover o desenvolvimento de crianças com paralisia cerebral. Educação e Pesquisa, São Paulo, v. 36, n. especial, p. 133-143, 2010. Disponível em: < http://www.scielo.br/pdf/ep/v36nspe/v36nspea11.pdf>. Acesso em: 17 jul. 2014.

; PAZ JÚNIOR, A. C. Método Sarah: reabilitação baseada na família e no contexto da criança com lesão cerebral. São Paulo: Ed. Santos, 2008.

BRONFENBRENNER, U. Bioecologia do desenvolvimento humano: tornando os 
seres humanos mais humanos. São Paulo: Artmed, 2011.

BUENO, J. G. S. Educação especial brasileira: integração/segregação do aluno diferente. São Paulo: EDUC, 2004.

CARVALHO, M. B. W. B. Política de educação especial: o acesso à escola e a responsabilidade do poder público. In: CABRAL NETO, A.; NASCIMENTO, I. V.; LIMA, R. N. (Org.). Política pública de educação no Brasil: compartilhando saberes e reflexões. Porto Alegre: Sulina, 2006.

CATROPPA, C.; ANDERSON, V. Traumatic brain injury in childhood: rehabilitation considerations. Developmental Neurorehabilitation, United Kingdom, v. 12, n. 1, p. 5361, fev. 2009. Available in: < http://www.tandfonline.com/doi/full/10.1080/17518420802634476>. Acess in: 15 jul. 2014.

DURAN, R.; GOODMAN, R. Morbidade psiquiátrica em crianças com alterações neurológicas. Rev. Bras. Psiquiatr., São Paulo, v. 22, supl. 2, p. 52-54, dez. 2000. Disponível em: <http://www.scielo.br/pdf/rbp/v22s2/3799.pdf $>$. Acesso em: 30 jun. 2014.

FEENEY, T.; YLVISAKER, M. Context-sensitive cognitive-behavioural supports for young children with TBI: a replication study. Brain injury, United Kingdom, v. 20, n. 6, p. 629-645, jun. 2006. Available in: $<$ http://www.tandfonline.com/doi/abs/10.1080/02699050600744194?journalCode $=$ ibij2 0). Acess in: 12 dez. 2014.

O desempenho escolar após a lesão cerebral: questões comportamentais, sociais e acadêmicas. In: BRAGA, L.; PAZ JÚNIOR, A. C. Método Sarah: reabilitação baseada na família e no contexto da criança com lesão cerebral. São Paulo: Santos, 2008.

FORMAN, M. A família da criança com lesão cerebral. IN: BRAGA, L.; PAZ JÚNIOR, A. C. Método Sarah: reabilitação baseada na família e no contexto da criança com lesão cerebral. São Paulo: Santos, 2008.

JANNUZZI, G. M. A educação do deficiente no Brasil: dos primórdios ao início do século XXI. Campinas: Autores Associados, 2006.

MAZZOTTA, M. Educação especial no Brasil: história e políticas públicas. São Paulo: Cortez, 2005.

PRIGATANO, G.; GRAY, J. Parental concerns and distress after paediatric traumatic brain injury: a qualitative study. Brain injury, United Kingdom, v. 21, n. 7, p. 721-729, jun. 2007. Available in: <https://www.ncbi.nlm.nih.gov/pubmed/17653946>. Acess in: 10 dez. 2015.

REDE SARAH. Informações sobre doenças tratadas. Disponível em: < www.sarah.br $>$. Acesso em: 10 dez. 2015.

SOUZA, J.; KANTORSKI, L. P.; LUIS, M. A. V. Análise documental e observação participante na pesquisa em saúde mental. Revista Baiana de Enfermagem, Salvador, v. 
Dossiê: Diálogos interdisciplinares em Psicologia da Educação

|A interdisciplinaridade entre Psicologia Hospitalar, reabilitação e educação no trabalho com crianças com lesão cerebral|

| Carolina Alves Rezende Alcântara |

25, n. 2, p. 221-228, maio/ago. 2011. Disponível em: $<$ https://portalseer.ufba.br/index.php/enfermagem/article/view/5252/4469>. Acesso em: 15 jul. 2014. 